\title{
Developments Towards Imaging Nanoscale Biology with XFELs: Some Recent Examples and a Glance to the Future.
}

$\underline{\text { Adrian P. Mancuso }}^{1,}$,, Andrew Aquila ${ }^{2}$, Richard Bean ${ }^{1}$, Johan Bielecki ${ }^{1}$, Carsten Fortmann-Grote ${ }^{1}$, Klaus Giewekemeyer ${ }^{1}$, Yoonhee Kim ${ }^{1}$, Ruslan Kurta ${ }^{1}$, Romain Letrun ${ }^{1}$, Masoud Mehrjoo ${ }^{1, a}$, Marc Messerschmidt ${ }^{1,3}$, Grant Mills ${ }^{1,4}$, Adam Round ${ }^{1,5}$, Tokushi Sato ${ }^{1,6}$, Joachim Schulz ${ }^{1}$, Marcin Sikorski ${ }^{1}$, Stephan Stern ${ }^{1,6}$, Patrik Vagovic ${ }^{1,6}$ and Thomas Tschentscher ${ }^{1}$

1. European X-ray Free Electron Laser Facility GmbH, Holzkoppel 4, 22869, Schenefeld, Germany

2. SLAC National Accelerator Laboratory, 2575 Sand Hill Road, Menlo Park (CA) 94025, USA

3. BioXFEL Science and Technology Center, Buffalo, NY, USA

4. ARC Centre of Advanced Molecular Imaging, Department of Chemistry and Physics, La Trobe Institute for Molecular Science, La Trobe University, Victoria 3086, Australia

5. Faculty of Natural Sciences, Keele University, Staffordshire, ST5 5BG, UK

6. Center for Free Electron Laser Science, Deutsches Elektronen-Synchrotron, Notkestraße 85, 22607 Hamburg, Germany

a. Now at Deutsches Elektronen-Synchrotron, Notkestraße 85, 22607 Hamburg, Germany

* Corresponding author, adrian.mancuso@xfel.eu

The relatively recent advent of extremely high brightness X-ray sources - such as X-ray Free Electron Lasers (XFELs) - has made it possible to consider using such X-rays to investigate the structure of noncrystalline, biological matter at ever improving resolutions [1]. The promise of such investigations opens the possibility of studying biologically relevant proteins or viruses that - due to the difficulty or absence of their crystallization - may not be studied with conventional methods of structural biology. One goal is atomic resolution structure determination of such biologically relevant, non-crystalline (and indeed perhaps never crystalline) systems [2].

The key idea is to illuminate a series of identical or near identical particles with the bright $\left(>10^{12}\right.$ photons per pulse) and short (durations of femtoseconds) pulses of XFEL radiation, collect the resultant two-dimensional diffraction from many (tens to hundreds of thousands) of realizations and compose that data into a three-dimensional (3D) diffraction data volume, which can in turn be inverted into the 3D structure of the sample in question [3]. The method is still relatively novel, and to date, a great deal of work has been undertaken to drive the method from first proof-of-principle [4], and early examples [5,6] though reconstructions of viruses in $3 \mathrm{D}[7,8]$.

We have recently undertaken research work that further supports the methodological development of single particle imaging (SPI) with X-ray Free Electron Lasers. Herein we briefly describe four different facets of that work, as well as providing an outlook to what the future might hold in light of the recent operation of the very high repetition rate European XFEL [9] in the Hamburg metropolitan region of Germany.

It is expected that such SPI experiments produce very weak diffraction data (perhaps of order one hundred photons per frame) from single particles that are of biological interest. An open question is, for how small a particle and to what resolution can one practically make a three-dimensional structure? To explore this question, we have developed a detailed modeling workflow that accounts for much of the physics in such an experiment including the properties of the incident XFEL beam, the photon-matter 
interaction between the intense XFEL beam and a sample, the diffraction to a detector and more [10-12]. This rather sophisticated model has allowed us to systematically explore some of the parameter space relevant to SPI. One such case study involved exploring the effect of pulse duration (and hence degree of radiation damage) on a model system and ascertaining that, for the parameters modeled, the optimal pulse duration was about 9 fs [12].

In a further attempt to push the boundaries of the SPI methods, we have performed an analogue of an SPI experiment using synchrotron radiation. A larger $(\sim 1 \mu \mathrm{m})$, more scattering sample was illuminated with coherent X-rays using exposure times in the millisecond range allowing about one hundred (100) scattered photons to be detected per frame of data. This is similar to the signal level expected in an SPI experiment at an XFEL with a small $(\sim 100 \mathrm{~nm})$, non-crystalline biological sample [13]. We have performed analysis on many $(>100000)$ diffraction patterns from such an experiment and quantify the fidelity of the resultant reconstructed three-dimensional electron density.

In an alternative work led by Kurta and colleagues [8], a different approach to determining the 3D structure of non-crystalline particles using correlations has been performed. Using data collected from two different sub-100 nm diameter viruses at the Linac Coherent Light Source (LCLS) in the USA, Kurta and colleagues used methods of angular correlations [14] to calculate correlation functions, which can in turn be inverted to yield the 3D structure of the virus. Importantly, the method showed that the two viruses studied have structure that differs from an ideal icosahedral symmetry, demonstrating the power of the method to determine structures in the absence of symmetry assumptions.

Another important parameter in coherent diffractive imaging is the nature of the wavefront incident on the sample. In the simplest cases, the wavefront is either effectively a plane wave, or assumed to be plane. In practice, for a focused beam that may be similar in transverse size to the sample under investigation, this assumption likely does not hold true. Furthermore, the SASE nature of an FEL beam more or less ensures that each pulse of FEL radiation exhibits variation in a number of parameters, including wavefront. Nevertheless, the knowledge of the wavefront incident on the sample is in principle sufficient to remove its influence in subsequent analysis, though this requires a non-invasive, shot-byshot wavefront determination technique. We have performed a study using such a technique at the Free electron LASer in Hamburg (FLASH), primarily to observe and quantify the wavefronts of individual FEL pulses and characterize their variations both shot-to-shot and for different configurations of the FLASH accelerator [15].

In the not too distant future, the scope for SPI-type experiments at hard X-ray FELs will improve greatly. Recently the SPB/SFX instrument of the European XFEL [16] has come online. This instrument is designed to capitalize on the high repetition rate of the European XFEL making experiments that are presently difficult, for reasons of insufficient data volume at lower repetition rate FELs, much more viable. Further improvements in sample delivery [17] and tailored focusing optics [18] as well as low background experiment design [16] and detectors [19] are expected to further improve the conditions for such measurements and interpretation of very weak diffraction signal from weakly scattering, noncrystalline biological materials [20].

\section{References:}

A. Aquila, et al, Struct. Dyn. 2, 041701 (2015).

M. Altarelli and A. P. Mancuso, Philosophical Transactions of the Royal Society of London 
[3] R. Neutze, R. Wouts, D. van der Spoel, E. Weckert, and J. Hajdu, Nature 406, 752 (2000).

[4] H. N. Chapman, A. Barty, M. Bogan, S. Boutet, M. Frank, S. Hau-Riege, S. Marchesini, B. Woods, S. Bajt, and W. Benner, Nature Physics, 2, 839 (2006).

[5] A. P. Mancuso, O. M. Yefanov, and I. A. Vartanyants, Journal of Biotechnology 149, 229 (2010).

[6] M. Marvin Seibert, et al, Journal of Physics B: Atomic, Molecular and Optical Physics 43, 194015 (2010).

[7] T. Ekeberg, et al., Phys. Rev. Lett. 114, 098102 (2015).

[8] R. P. Kurta, et al, Phys. Rev. Lett. 119, 237 (2017).

[9] T. Tschentscher, et al Applied Sciences, 7(6), 592-35 (2017).

[10] C. H. Yoon, et al, Sci. Rep. 1 (2016).

[11] C. Fortmann-Grote, et al, arXiv Preprint arXiv:1610.05980 (2016).

[12] C. Fortmann-Grote, et al, IUCrJ 4, 560 (2017).

[13] K. Giewekemeyer, et al, in preparation.

[14] Z. Kam, Macromolecules 10, 927 (1977).

[15] M. Mehrjoo, et al, Opt Express 25, 17892 (2017).

[16] A. P. Mancuso, et al, Technical Design Report Scientific Instrument Single Particles, Clusters, and Biomolecules (SPB) (2013). doi:10.3204/XFEL.EU/TR-2013-004

[17] M. F. Hantke, Nature Photonics 8, 943 (2014) \& M. F. Hantke, et al, submitted.

[18] R. J. Bean, et al, Journal of Optics 18, 074011 (2016).

[19] D. Greiffenberg, J Instrum 7, C01103 (2012).

[20] The authors acknowledge the engineering and technical contributions of the SPB/SFX engineers and technicians, namely Thomas Dietze, Alexis Legrand, Felix Lemcke, Luis Lopez, Bradley Manning, Nadja Reimers, Philipp Schütt, Cedric Signe Takem, Andrew Stawniczy and Prasad Thute. The SFX User Consortium is thanked for providing valuable input as well as part of the funding for the SPB/SFX instrument. This project has received funding from the European Union's Horizon 2020 research and innovation programme under grant agreement No 654220. Funding has also been provided by the NSF STC Award 'BioXFEL' (1231306).

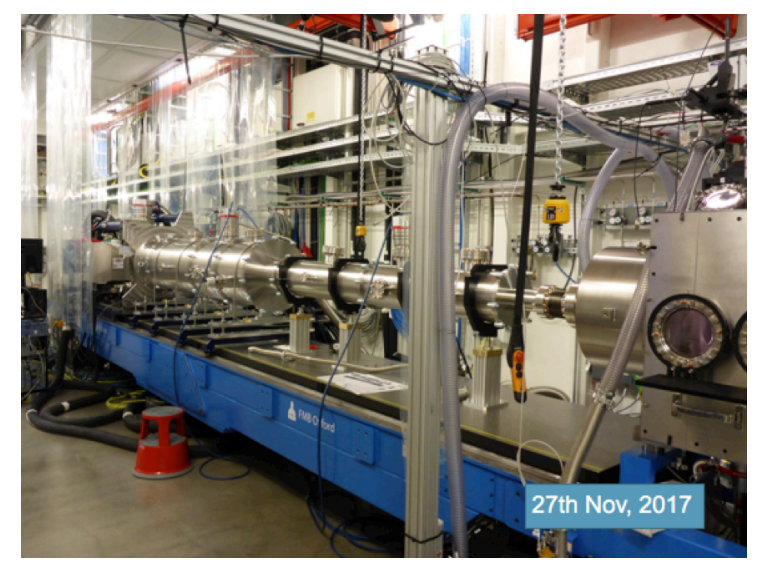

Figure. 1. Photograph of the SPB/SFX instrument of the European XFEL set up in "long" ( $\sim 5.5 \mathrm{~m})$ sample to detector geometry for single particle imaging of $\sim 500 \mathrm{~nm}$ diameter samples. 
https://doi.org/10.1017/S143192761801276X Published online by Cambridge University Press 